\title{
Portable Microscopes for Scientific Publications
}

\section{Andrew Monk}

ioLight Limited, The Director General's House, 15 Rockstone Place, Southampton SO15 2EP

andrew.monk@iolight.co.uk

\begin{abstract}
This article reviews the use of portable microscopes in published science from Antonie van Leeuwenhoek's letters to the Royal Society to a recent paper on the phylogeny of the phorid fly by Hartop et al. [1]. The portability of a pocket microscope is a clear benefit, yet bulky compound microscopes remain the mainstay of field research and diagnosis. There is a growing interest in the impact of climate change, which drives researchers to investigate the smallest, most fragile life in remote places such as Antarctica, Mount Everest, and the Amazon Rainforest. Are pocket microscopes ready to produce publication-standard images?
\end{abstract}

Keywords: portable microscopy, climate change, field research, citizen science, point of care

\section{The Evolution of the Microscope}

Antonie van Leeuwenhoek is regarded by many as the father of microbiology. Working in seventeeth-century Delft, he was the first person to produce beautifully detailed drawings of protozoa, spermatozoa, bacteria, red blood cells, and many other biological subjects that modern microscopists observe every day.

Van Leeuwenhoek's microscopes (Figure 1) used a single ball lens made using a technique that was not replicated until 1957. The sample was mounted on a pin in front of the lens, with three screws to adjust its $X, Y$, and $Z$ position. These were small, highly portable instruments that could be taken anywhere, allowing their user to look at and draw previously unknown microscopic organisms. It was the portability of van Leeuwenhoek's tools that allowed him to be so prolific.

At the same time, other early microscopists were improving microscope designs to give a higher magnification, a wider field of view and fewer aberrations. Galileo turned his telescope around and was able to focus on small objects close to the lens, but this microscope was 2 meters long. So now the race was on to develop the compound microscope that we see in every microbiology laboratory and classroom today.

Modern compound microscopes are wonderful instruments. They give us clear images of subjects as small as $1 \mu \mathrm{m}$ across a wide field of view with minimal aberrations. These instruments are at the heart of modern microbiology and many other sciences. Microscopists have added dark-field, phase contrast, and polarized light illumination and cameras. We have inverted the microscope, and used electrons, confocal lasers, and many other techniques to increase resolution to below the wavelength of light. Antonie Van Leeuwenhoek would have been mightily impressed. Perhaps, however, he would have missed the portability that allowed him to take his simple microscope wherever he went.

Modern scientists are trained to rely on primary observation whenever possible. To a biologist this means observing the sample in the field as close as possible to its natural habitat, just as van Leeuwenhoek did. Removing a sample from its environment inevitably changes it with different temperature, humidity, and light conditions. It also means that field scientists must wait until they get back to the lab for their observations and carry a sample bag back from remote sites. Until recently, this made it difficult to carry out field research very far from a laboratory. This is a growing problem at a time when climate change makes remote field research ever more important as we explore its impact on the smallest, most fragile organisms in the toughest environments.

John McArthur took up the portable microscope challenge in the 1930s. The McArthur microscope (Figure 2) is much loved and still available today. It produces excellent images, though it is a little fiddly to use. It is an inverted microscope with the light path folded to an eyepiece looking downward. The slide is manipulated on a small stage, and the objective lenses can be selected to give the best magnification. The microscope is easiest to use on a tripod, leaving hands free to focus the microscope and move the slide.

In 2016, the British start-up ioLight introduced a self-contained pocket microscope (Figure 3) that displays its image on a mobile phone screen ready for instant sharing. The microscope sits on any stable surface, making it simple to focus and manipulate the slide.

\section{Magnification with Digital Microscopes}

The specification of digital microscopes can be rather confusing. Microscopes are advertised online at pocket money prices with magnifications of $\times 1000$ or more. How can this be when microscopists are used to paying the price of a car for an optical microscope of this specification and then having to use oil immersion to get $\times 1000$ ? The answer is that digital imaging allows the user to enlarge any image hundreds of times, but the magnification tells you nothing about the quality of the image. To understand this, you need to know the resolution of the instrument. Scientists may argue long into the night about the technical definition of resolution, but a good, practical definition is that a microscope claiming a resolution of $1 \mu \mathrm{m}$ should be able to resolve $1 \mu \mathrm{m}$-wide black lines that are separated by $1 \mu \mathrm{m}$. The image should show distinct lines rather than a black blob. The resolution of optical microscopes depends on the quality of the lenses and how well set up the instrument is, but a resolution of $0.5 \mu \mathrm{m}$ is typical of a good microscope. Conventional optical microscopes are limited to resolutions of approximately the wavelength of light. A resolution of $1 \mu \mathrm{m}$ is ideal for observing features of $5 \mu \mathrm{m}$ or larger including animal and plant cells, algae, protozoa, and parasites.

\section{Climate Change}

The recent interest in field microscopes has been driven by the huge surge in climate change research. Biologists across disciplines are analyzing the impact climate change has on a wide range of ecosystems.

In 2019 National Geographic ${ }^{\circledR}$ measured the impact of climate change on Mount Everest. Paul Mayewski, Expedition Leader and Chief Scientist, said, "Once you get to about 5,000 m, you are above where most of the science on the planet has been done. Between 5,000 $\mathrm{m}$ and the top of Everest, there has been almost nothing." [2]

Monitoring protozoa in these conditions is extremely difficult, and it is becoming increasingly urgent for scientists to 


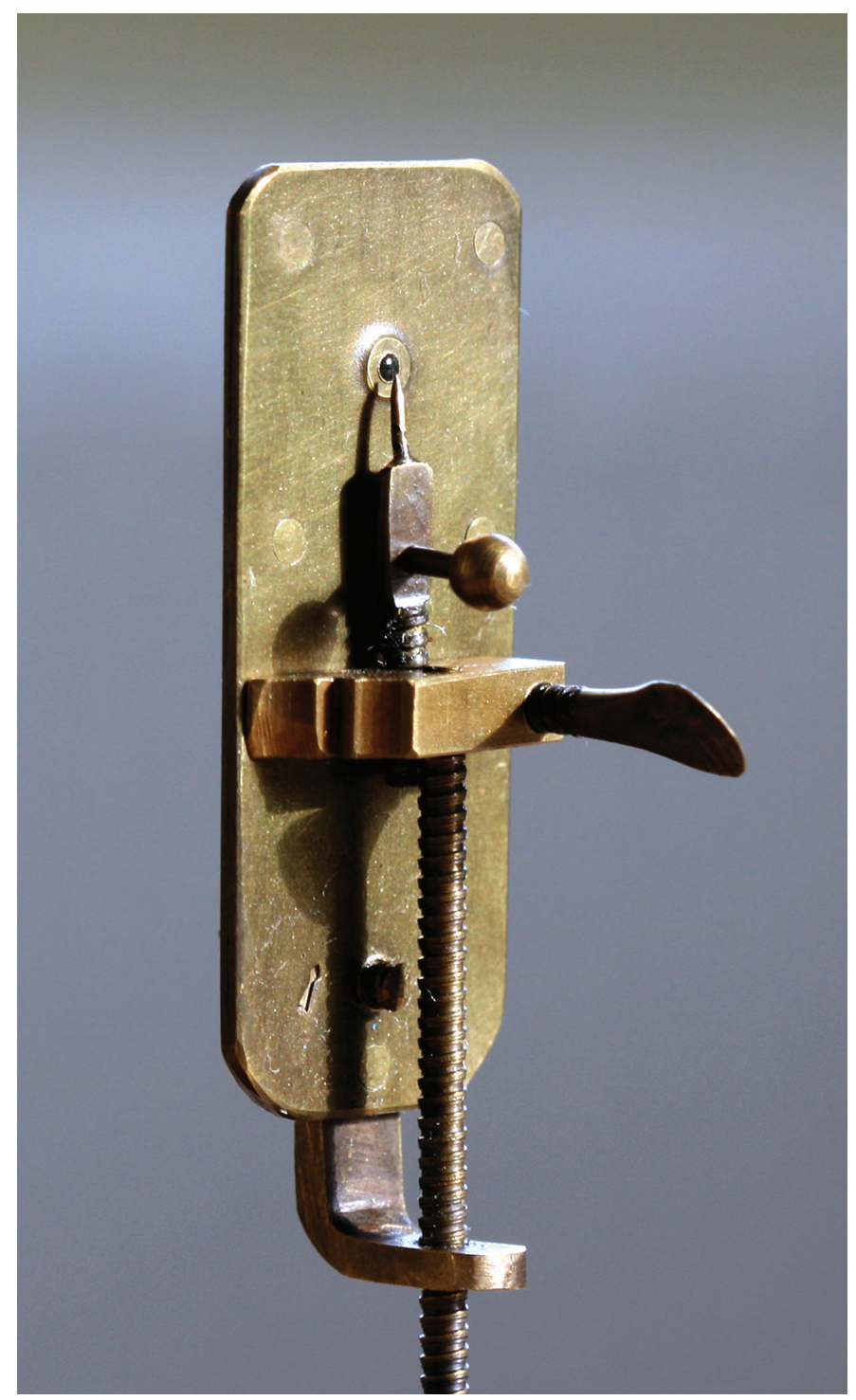

Figure 1: Antonie van Leeuwenhoek's microscope. Image by Jeroen Rouwkema. Image reproduced from https://en.wikipedia.org/wiki/Antonie_van_ Leeuwenhoek\#/media/File:Leeuwenhoek_Microscope.png, used under a Creative Commons Attribution (CC BY-SA 3.0) license.

access a microscope as portable as van Leeuwenhoek's with the performance of a modern laboratory microscope. The National Geographic ${ }^{\circledR}$ Perpetual Planet Expedition to Everest used an ioLight microscope with a resolution of $1 \mu \mathrm{m}$.

\section{Citizen Science versus Algal Blooms}

One benefit of portable digital microscopes is that they are much easier to use than their laboratory cousins and can be used effectively with very little training. Mobile phone experience is as useful as microscopy experience, which makes them a perfect fit for young scientists and a useful tool in engaging them in scientific research. This is a huge benefit in science outreach, where compound microscopes are difficult to use and get in the way of the science. Digital microscopes, on the other hand, are second nature to young scientists and, perhaps, even cool. Inspiring young people in science is crucially important to a society learning how to manage climate change and pandemics.

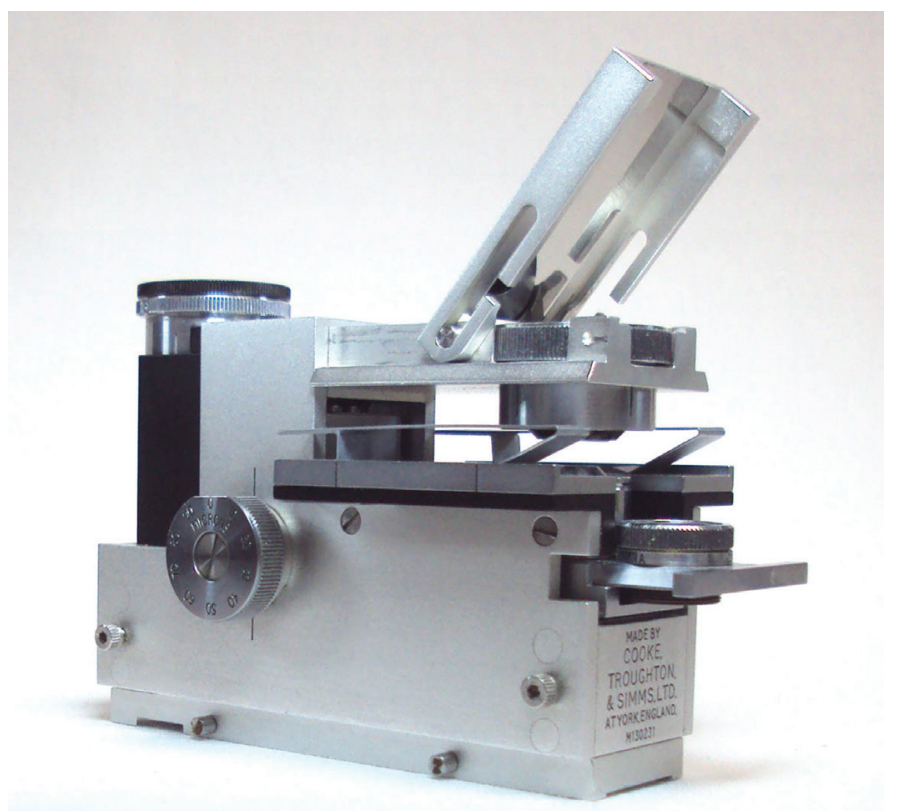

Figure 2: 1960s McArthur Microscope. Courtesy of microscope-antiques.com.

Climate change and chemical farming are two of the causes of algal blooms, providing a nutrient-rich soup in which algae thrive. Some species of these algae produce toxins that kill fish, marine animals, dogs, and potentially humans. The Centre for Environment, Fisheries and Aquaculture Science (CEFAS) is responsible for monitoring the impact of harmful algal blooms (HAB) on the European coastline. Dr. Andy Turner of CEFAS said, "We are working with the Five Islands Academy School on St. Mary's, and other people on the Isles of Scilly including children, ex-pupils and teachers to collect and process water samples." Dr. Turner continued, "Our lead analyst in the islands, Zoe Jenkins, is studying biology in her final year at Aberystwyth University. Zoe was unable to leave her home on St. Mary's. However, even with COVID-19 restrictions in place, we hope that citizen monitoring using portable digital microscopes will generate new science when normal laboratory-based approaches are not possible. CEFAS is getting

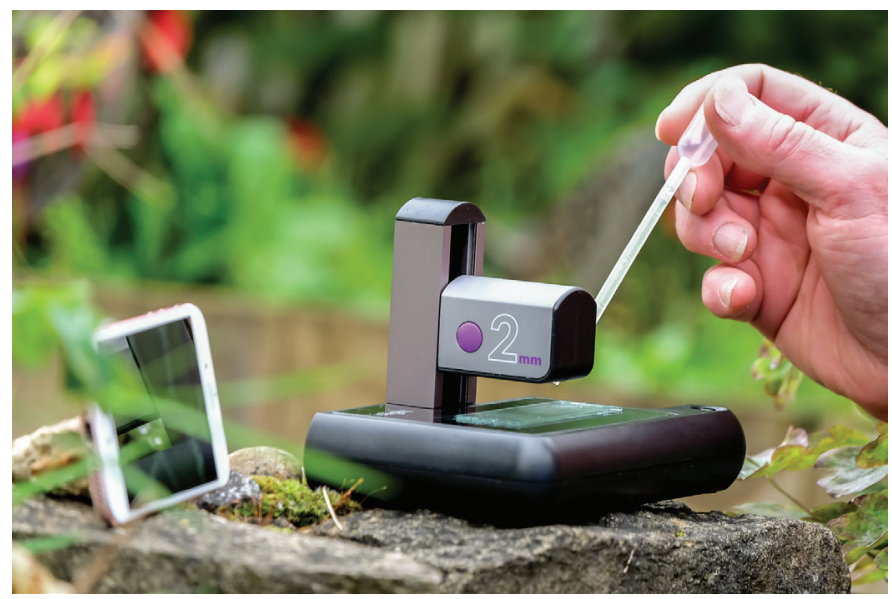

Figure 3: ioLight's portable microscope. 


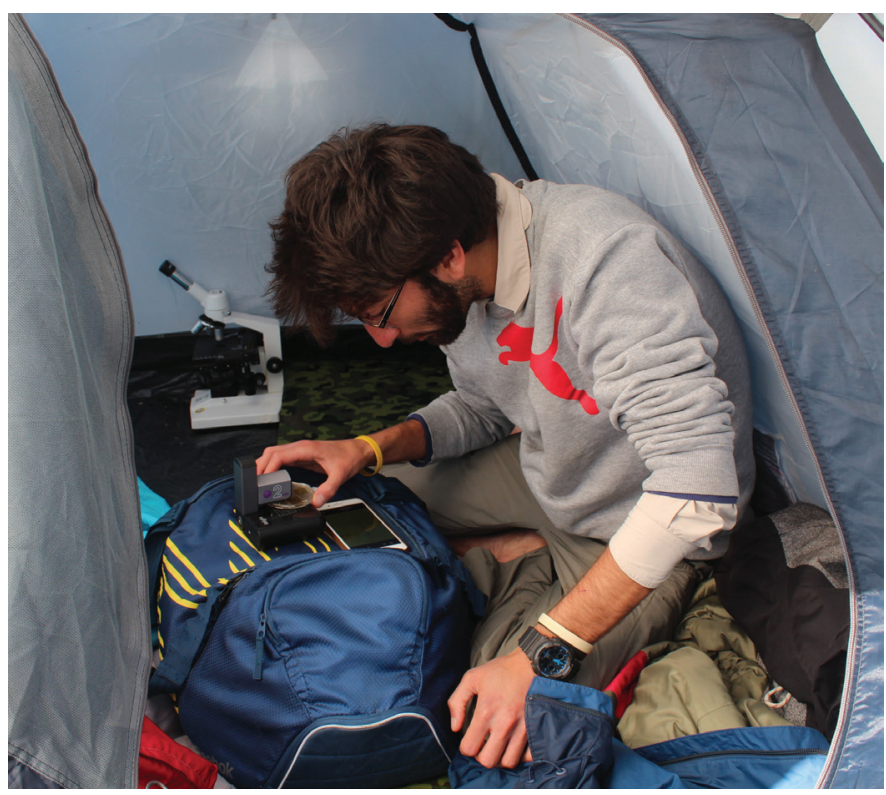

Figure 4: Munib Khanyari's Kazakhstan field laboratory (broken traditional microscope shown in the background).

real-time information on algal blooms, and people in The Scilly Isles are learning about their precious environment."

Just across the Atlantic Ocean from the Isles of Scilly, the State of New York (NY) has approximately 8,000 lakes managed by 200 Lake Associations. These are made up of residents living on the shores of the lake who have a strong interest in maintaining the health of their lake. Suspect algal blooms are reported to the NY Department of Environmental Conservation, which may issue an advisory notice closing the lake to recreational activities.

Professor Greg Boyer of the State University of New York (SUNY) College of Environmental Science and Forestry (ESF) said that many of these blooms are in fact harmless, but diagnosis requires a microscope for visual identification. Lake Association members often drive long distances to bring samples to the lab in Syracuse, NY to have their samples checked to ensure their lake is safe. The ESF team is trialling a portable microscope, which allows Lake Associations to capture highresolution microscope images of the algae on their mobile phones and instantly send them to ESF for analysis. This saves both time and expense and gives the Lake Associations immediate access to this important information.

\section{Saving the Saiga}

In 2015 at least 100,000 Saiga antelope died in Kazakhstan of a strange epizootic disease-the animal equivalent of an epidemic. The Royal Veterinary College coordinated a major research collaboration to uncover the cause of this sudden increase in Saiga mortality.

Munib Khanyari of Bristol University described working conditions in the field: "Working in remote and harsh locations such as the Kazakh Steppe or the Indian Trans-Himalayas means doing field work many kilometres away from basic necessities and comfort. It is a logistical nightmare to store and safely transport samples, frequently resulting in their contamination or loss. Often it is difficult to obtain permits to transport samples out of the study area. In such cases having a portable microscope enables in-field analysis of samples, improving efficiency and ease of robust science." His colleague Hannah Vineer added, "When working in extreme weather conditions samples can perish quickly. I have found having a portable microscope with image capture really useful to get a second opinion. It also came in handy when our main laboratory microscope fell apart as soon as we arrived at our camp in Kazakhstan after several bumpy days on the road to reach the site!" (Figure 4)

\section{Portable Microscopes for Published Science}

We have seen how useful modern portable microscopes are for public outreach, citizen science, and remote fieldwork. However, Antonie van Leeuwenhoek is credited with the discovery of protists, bacteria, spermatozoa, and many other microscopic organisms that had never been seen by human eye. Modern digital portable microscopes have understandably earned a bad press because of their exaggerated magnification claims. Are they really good enough for serious science?

Emily Hartop et al. recently published the first peerreviewed paper to feature images captured on a portable microscope [3]. The paper, "Scuttling towards monophyly: phylogeny of the mega-diverse genus Megaselia (Diptera: Phoridae)," takes a deep dive into this complex phylogeny using both molecular and morphological analyses. The team worked in the field with a portable microscope, and the paper features images of the specimens' setae. These illustrate the morphological differences between three of the groups of Megaselia identified: the spinigera group, the ruficornis group, and the "core" Megaselia. The paper was published in September 2020 in Systematic Entomology, a Royal Entomological Society journal, and represents the largest study to date of Megaselia relationships based on molecular data.

Lead analyst, Emily Hartop, is a keen citizen science advocate and regularly travels between Europe, the U.S., and Singapore to engage with collaborators and work on numerous projects. Hartop needed a compact portable microscope to support her preliminary phylogenetic analysis of Megaselia and ongoing research. With a portable microscope in her bag, she was able to analyze specimens everywhere from her living room to a laboratory on the other side of the world. She captured important morphological features and Z-stacked the images to achieve a detailed 3D depiction of the phorid flies (Figure 5).

The Megaselia genus is notoriously challenging to study due to its extreme species diversity, limited knowledge of higher-level relationships, and lack of molecular data. The recent scientific study provides a framework for future work that Hartop hopes will determine how big the Megaselia genus is, in terms of the number of species, their life history, and habitats; the spatial and temporal distributions of the species; and the different evolutionary clades.

"Democratising science is hugely important. If people around the world are unable to do the same science that we do in countries that are more fortunate, then that is limiting science significantly. Portable microscopes can be a big part of helping to change this," explained Hartop. "I didn't set out to be the first scientist to publish a peer-reviewed journal article with digital images from a portable microscope, but that's exciting because it shows that these instruments are perfectly 


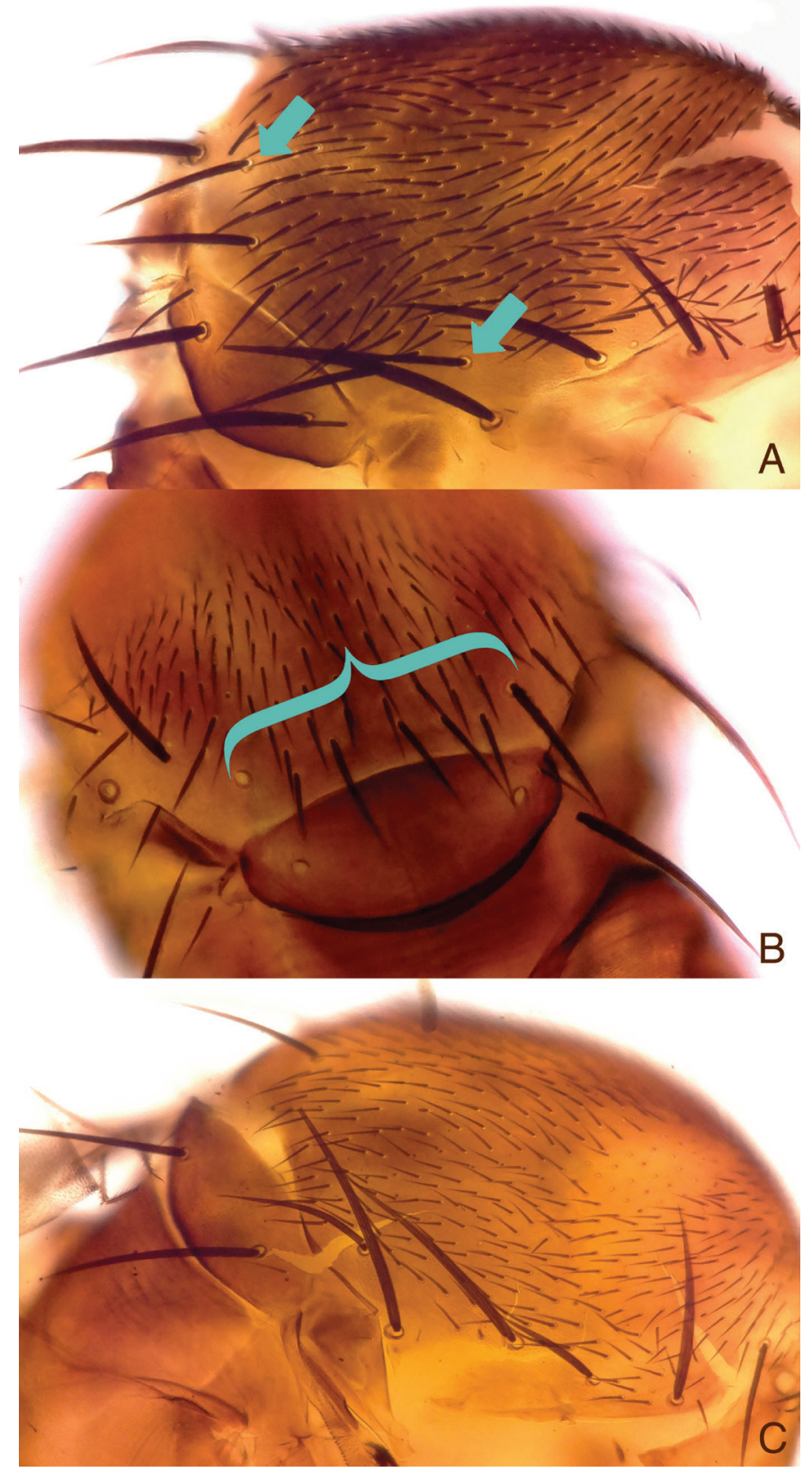

Figure 5: Z-stacked images of Megaselia setae from Reference 1.

capable of creating quality images suitable for publication in respected journals."

\section{What Next for Portable Microscopes?}

Having proved their value in public outreach, citizen science, fieldwork, and now published science, what is next for portable microscopes?

The microscope is the gold standard for most diagnostic protocols for pathology in humans, animals, fish, and crops, as well as many engineering diagnostic protocols such as detection of asbestos. The potential for taking these tests out of the laboratory and delivering them literally anywhere on the planet is really exciting. In wealthy countries, nobody is very far from a microbiology lab, and tests can be carried out for any disease quickly and with high reliability. However, in

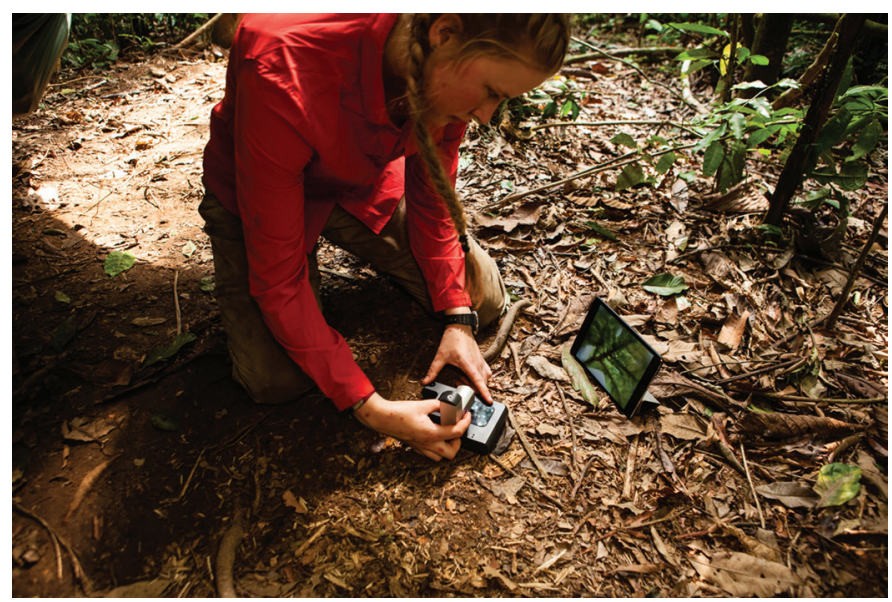

Figure 6: Portable microscope being used in the Amazon Rainforest.

less privileged communities, the state often cannot afford this infrastructure. Even if it can, there are difficulties in transporting large numbers of samples to a handful of labs. Where this is done successfully, it is still difficult to match a diagnosis to a person and then prescribe a treatment.

For example, The World Health Organization (WHO) said, "Schistosomiasis (Bilharzia) affects almost 240 million people worldwide, and more than 700 million people live in endemic areas. The infection is prevalent in tropical and subtropical areas, in poor communities without potable water and adequate sanitation" [3] such as the Amazon Rain Forest (Figure 6). The disease is caused by a blood fluke and detected by counting parasite eggs in fecal smears with a microscope. The WHO aims to eradicate schistosomiasis and coordinates a wide test and treatment program across endemic areas. Teams visit schools to test for schistosomiasis, lymphatic filariasis, malaria, and other major killers.

Portable microscopes already offer a simple, robust method of capturing diagnostic images to inform treatment and epidemiology. However, the future is even more exciting. The images produced by inexpensive microscopes are ideal for processing by image recognition software that is already available. This raises the potential of automating medical diagnosis in remote locations so that it can be carried out by a competent technician with a very fast turnaround and a high precision. There is more work to do to develop the mobile test platforms, but portable microscopes bring us one step closer to eradicating these neglected tropical diseases (NTDs) that prevent remote communities from generating wealth and lifting themselves out of poverty.

Perhaps we have at last got back to a microscope that Antonie van Leeuwenhoek would enjoy.

\section{References}

[1] E Hartop et al., Syst Entomol (Sept. 2020) 1-12.

[2] Inside the Perpetual Planet Expedition to Mount Everest (video) (2020), National Geographic Society. www. nationalgeographic.org/media/inside-perpetual-planetexpedition-mount-everest.

[3] Schistosomiasis (Bilharzia), World Health Organization. www.who.int/health-topics/schistosomiasis\#tab=tab_1. 